\title{
Hábitos de consumo en plataformas e-commerce en adultos jóvenes de la ciudad de Bogotá
}

\author{
James Paul Linero Bocanegra* \\ Luis Fernando Botero Cardona*
}

Fecha de recibido: 25 de agosto de 2019

Fecha de aprobado: 16 de septiembre de 2019

Para citar: Linero Bocanegra, J. P. \& Botero Cardona, L. F. (2020). Hábitos de consumo en plataformas e-commerce en adultos jóvenes de la ciudad de Bogotá. Revista Universidad E Empresa, 22(38), 211-236. https://doi.org/10.12804/revistas.urosario.edu. co/empresa/a.8131

* Profesional en Comercio Internacional y Magíster en Relaciones Internacionales. Docente Investigador, Facultad de Mercadeo. Universidad Santo Tomás. Bogotá, Colombia. Correo electrónico: james.linero@gmail.com

** Profesional en Ingeniería de Sistemas y Magíster en Administración de Empresas. Docente Investigador, Facultad de Mercadeo. Universidad Santo Tomás. Bogotá, Colombia. Correo electrónico: fernando.l.botero@hotmail.com 


\section{Resumen}

El consumo en plataformas e-commerce está determinado por variables que afectan la confianza en línea para la toma de decisión de compra. El presente artículo divulga los resultados obtenidos a partir de una investigación cualitativa, que tuvo por objetivo la identificación de los hábitos de consumo en línea, en una muestra de personas entre los 24 y 34 años, de estratos socioeconómicos 3 y 4 de la ciudad de Bogotá. Según los resultados, dicha confianza está asociada, principalmente, con la información suministrada por el sitio web y con su historial de comentarios, la calidad del servicio posterior a la transacción en línea, la seguridad percibida y la utilidad generada en el uso de dicha plataforma. El impacto generado por el comercio electrónico en Colombia conlleva a la necesidad de investigaciones sobre las variables que definen las conductas de compra de los consumidores y que permiten una mayor efectividad en la implementación de estrategias de e-commerce en las compañías.

Palabras clave: E-commerce, hábitos de consumo en línea, confianza en línea.

\section{Consumer habits in e-commerce platforms in young adults in Bogotá}

\section{Abstract}

Consumption in e-commerce platforms is determined by variables that affect online trust for purchase decision making. The present article discloses the results obtained from qualitative research, aimed at identifying online consumption habits, in a sample of people between 24 and 34 years of age, from socio-economic levels 3 and 4 in Bogotá city. According to the results, clients associate trust mainly with the information provided by the website and its comments history, the quality of the service after the online transaction, the perceived security, and the utility generated in the use of a given platform. The impact generated by electronic commerce in Colombia leads to the need for researches on the variables that define consumers'purchasing behavior and that allow greater effectiveness in the implementation of e-commerce strategies in companies.

Keywords: E-commerce, online consumer habits, online trust.

\section{Hábitos de consumo em plataformas e-commerce em adultos jovens da cidade de Bogotá}

\section{Resumo}

O consumo em plataformas e-commerce, está determinada por variáveis que afetam a confiança online para a tomada de decisão de compra, o presente artigo divulga os resultados obtidos a partir de uma pesquisa qualitativa, que teve por objetivo a identificação dos hábitos de consumo online, em uma amostra de pessoas entre os 24 e 34 anos, de estratos socioeconômicos 3 e 4 da cidade de Bogotá. Segundo os resultados, dita confiança está associada principalmente à informação fornecida pelo site e o seu historial de comentários, a qualidade do serviço posterior à transação online, a segurança percebida e a utilidade gerada no uso de dita plataforma. O impacto gerado pelo comércio eletrônico na Colômbia, leva à necessidade de pesquisas sobre as variáveis que definem as condutas de compra dos consumidores e que permitem uma maior efetividade na implementação de estratégias de e-commerce nas companhias.

Palavras-chave: E-commerce, hábitos de consumo online, confiança online. 


\section{Introducción}

Frente a las tendencias y cambios acelerados en los hábitos de consumo generados por las nuevas tecnologías de la información y la comunicación, las compañías enfrentan la necesidad de orientar sus estrategias de e-commerce (Li, Quansheng \& Lin, 2017), de acuerdo con las variables de análisis que sustentan la confianza en línea (Kim \& Peterson, 2017) y, por ende, definen su conducta de compra y consumo.

Esta investigación se originó a partir de la formulación del siguiente interrogante: ¿cuáles son los hábitos, necesidades y prácticas de consumo en plataformas digitales e-commerce, en personas con edades entre los 24 y 34 , de los estratos 2,3 y 4 de la localidad Chapinero de la ciudad de Bogotá? Se abordó esta investigación desde una metodología de estudio etnográfico de tipo cualitativo (Bautista, 2011), utilizando la técnica de entrevista a profundidad, lo que permitió identificar los hábitos consumo del segmento estudiado respecto al uso de plataformas e-commerce, los resultados de la investigación fueron tratados a partir del análisis de redes semánticas usando la herramienta Atlas.ti v. 8.3.1.

Los resultados de esta investigación demostraron la importancia en las relaciones de las necesidades de los consumidores en línea, con respecto a la categoría de confianza en línea, definidos desde los hábitos de compra y consumo, y su relación con las dimensiones: tecnológica, económica, social y legal de los consumidores en línea. La comprensión de estas relaciones permitió conocer el grado de satisfacción y atributos valorados por los sujetos de estudio a partir transacciones en línea.

\section{Revisión de la literatura}

Según el World Economic Forum (2019), Colombia se encuentra liderando la red de Centros para la Cuarta Revolución Industrial que:

Reúne a gobiernos, compañías líderes, sociedad civil y expertos de todo el mundo para co-diseñar y probar enfoques innovadores en la política y gobierno de la tecnología. Su 
visión es dar forma al desarrollo y uso de la tecnología, de manera que se maximicen los beneficios y los riesgos se minimicen (World Economic Forum, 2019).

Así mismo, en Colombia, según el Consejo Privado de Competitividad 2018-2019, y de acuerdo con:

El índice de digitalización (Fundación Cotec para la innovación, 2016), Colombia ha logrado posicionarse en la "etapa avanzada" de su ecosistema digital, lo cual significa que el país ha conseguido avances considerables en infraestructura y acceso a tecnologías. En efecto, de acuerdo con los datos del Ministerio de Tecnologías de la Información y las Comunicaciones, durante los últimos diez años Colombia multiplicó por cuatro el porcentaje de personas que usa internet (Consejo Privado de Competitividad, 2018. p. 375).

Según el Informe Nacional de Competitividad 2018-2019:

Las conexiones de internet se multiplicaron por tres en los últimos cuatro años, logrando superar la meta de contar con 27 millones de conexiones de internet en el año 2018. Al cierre del primer trimestre de 2018, Colombia contaba con 30.383.982 de conexiones a internet de banda ancha (p. 376).

Sin embargo, Colombia aún está rezagada en comparación con países como Sudáfrica, México, Perú, Turquía, Chile, Argentina, Brasil, Malasia, Uruguay, Costa Rica y Corea del Sur. El mismo informe registra que: "en 12 departamentos del país la penetración de internet banda ancha fijo no alcanza a superar el 5\%, mientras que en Bogotá esa cifra asciende a $22.2 \%$ y en Antioquia y Risaralda a 16.6\%." (p. 377).

La importancia de la conectividad como base esencial del comercio electrónico también se encuentra soportada por The Global Competitiveness Index 2018, donde se señala que Colombia ocupa el puesto 60 de 140 países. Dentro de los pilares que se evalúa por el indicador, Colombia se ubica en el ranking 84 en el pilar sobre "Adopción de las Tics", con una calificación de 46.7. En ese mismo pilar, se registró en el puesto 46 en suscripciones de telefonía móvil, en el puesto 65 en suscripciones a internet fijo banda ancha, en el 
puesto 69 en suscripciones a internet de fibra óptica y en el puesto 74 con relación al número de usuarios de internet registrados (World Economic Forum, 2018, p. 163-165).

Por su parte, la Asociación Nacional de Empresarios de Colombia (ANDI) realizó un estudio denominado Encuesta de Transformación Digital 2017 a varias empresas afiliadas, que indicó según resultados, que:

Las principales barreras y desafíos que enfrentan hoy las compañías para lograr una transformación digital exitosa son, en su orden, falta de cultura (74.1\%), desconocimiento $(61.6 \%)$ y presupuesto $(56.3 \%)$. En la industria, actualmente, la principal preocupación es la falta de cultura (76.2\%), cuando en 2016, la principal preocupación fueron los recursos financieros. En el sector de servicios, las mayores barreras son el desconocimiento y la falta de cultura (71.4\%) (ANDI, 2018).

Adicionalmente, el Reporte de Industria Sector TIC 2017 de la Comisión de Regulación de Comunicaciones del Ministerio de las tics indica que el comercio se ha transformado por el uso de las plataformas digitales, para facilidad de negocios desde cualquier lugar del mundo. Actualmente, la comercialización de productos por medio de estas plataformas conlleva a la transformación, desde operaciones logísticas tradicionales, hacia una cadena de suministro digital, que generan mayor productividad y reducción de costos, así como el surgimiento de nuevos actores en los canales de distribución, beneficiando a sectores como comercio, transporte, turismo y telecomunicaciones (Comisión de Regulación de Comunicaciones, 2018).

Este panorama ha generado cambios en los hábitos tradicionales de compra en los colombianos, caracterizados principalmente por el rechazo y la desconfianza. De tal manera que la seguridad se convierte en un atributo determinante en la toma de decisiones y uso de las plataformas e-commerce, especialmente debido a situaciones como robo, pérdida de sus garantías, el no cumplimiento de promesas y demoras en la entrega de los productos adquiridos por plataformas e-commerce.

El e-commerce se encuentra soportado por el grado de confianza en línea provisto en los sitios web, logrado por parte de las compañías en las relaciones con el consumidor -(Business to Consumer - B2C)-. Kin \& Peterson (2017) identifican 16 relaciones de 
confianza en línea, en las cuales determinan los antecedentes de confianza en línea que incluyen la disposición a confiar, el riesgo percibido, la seguridad percibida, la privacidad percibida, la reputación percibida, la utilidad percibida, la calidad percibida del sistema, la calidad percibida de la información, la calidad percibida del servicio y la calidad percibida del diseño, los cuales generan los consecuentes de la confianza en línea investigadas por los autores que incluyen satisfacción, actitud, intención de compra, intención de compra re-compra, intención de usar el sitio web y lealtad.

Pavlou (2003) al respecto indica que las causas y consecuencias de la confianza en línea son estudiadas para determinar el grado de influencia ejercido sobre el consumidor y el grado en que esa confianza percibida por el consumidor influye en su intención de uso, y continuo uso del e-commerce en las páginas web.

Con respecto a la comprensión del consumidor en línea, es importante conocer previamente, el contexto de los intermediarios en línea, constituidos por las comunidades en línea y los productos en línea. Li, Quansheng \& Lin (2017) señalan que "los intermediarios en línea se han vuelto populares en los últimos años". Se identifican dos tipos de intermediarios en línea: la comunidad en línea (online community) y canales de producto (channel product). "Las comunidades en línea se definen como sitios web que contienen y comparten contenidos de comunicación, incluidas reseñas de productos y debates de los consumidores (por ejemplo, experiencias de compra, preferencias individuales, así como preguntas y respuestas)" (Li, Quansheng \& Lin, 2017, p. 191).

Los canales de productos se definen como sitios web que muestran y exhiben información de productos, que incorporan descripciones de productos y actividades de marketing por parte de los profesionales de marketing (por ejemplo, atributos clave de productos, actividades promocionales y publicidad persuasiva) (Li, Quansheng \& Lin, 2017).

Según los autores, las comunidades en línea y los canales de productos tienen dos diferencias principales, para los consumidores, las comunidades en línea sirven como lugar de comunicación gratuita con asesores para obtener información objetiva del producto, con los canales de producto no existe interacción. 
Por otro lado, para los profesionales del marketing, las comunidades en línea proporcionan enfoques para ejecutar las comunicaciones mutuas con los consumidores, mientras que el canal de productos es una herramienta para que transmitan información unidireccionalmente a los consumidores ( $\mathrm{Li}$, Quansheng \& Lin, 2017). Las comunidades en línea, influencian las decisiones de compra por las percepciones entre admiradores de una marca, lo que "permite a una organización establecer una serie de vínculos con sus consumidores" (Thompson \& Sinha, 2008).

A partir de lo anterior, el consumidor en línea es aquel que hace uso de las tecnologías de la información para adquirir productos por medio de plataformas web o aplicaciones en línea, en muchos casos, influenciados por las comunidades en línea, la confianza y diseño de las páginas web, las redes sociales y la percepción y lealtad de las marcas. "Cada vez más consumidores participan en plataformas en línea para interactuar con otros consumidores y con marcas" (Kelley \& Aalden, 2016, citados por Martínez, Anaya, Molinillo, Aguilar \& Esteban, 2017). Se trata entonces de realizar esfuerzos para comprender cómo crear y mantener comunidades, para aumentar el valor para el cliente (Martínez, Anaya, Molinillo, Aguilar \& Esteban, 2017). En muchos casos, es la forma como los mismos consumidores en línea realizan evaluación de los productos que desean comprar.

De esta manera, el estudio ofrece un acercamiento que permite identificar los hábitos del consumidor en línea generados a partir de las plataformas e-commerce en la población objeto de estudio, indicando las necesidades del consumo en línea motivadas por el uso de estas plataformas y describir sus prácticas de consumo en línea.

\section{Metodología}

La propuesta metodológica parte de un estudio etnográfico de tipo cualitativo (Bautista, 2011), por medio de la técnica de entrevista a profundidad que busca identificar los hábitos, necesidades y prácticas de consumo en plataformas e-commerce de personas con edades entre 24 y 34 años, ubicadas en la localidad de Chapinero de la ciudad de Bogotá. Todo esto, a partir de un muestreo de participantes voluntarios, a los cuales se les aplica un 
derrotero de preguntas desarrollado. Los resultados obtenidos son consolidados a partir de análisis de texto y discursos, mediante redes semánticas utilizando la herramienta Atlas.ti.

\section{Resultados}

El análisis de los datos para la categoría hábitos de consumo de las plataformas e-commerce presenta la relación entre el nivel de confianza en línea percibido y las actitudes y dimensiones del consumidor en línea, reflejadas en las prácticas de consumo mencionadas por los sujetos entrevistados.

La disposición de confianza es evidente en el entorno virtual de la página web y la experiencia posterior a la decisión de compra: "entonces como mi experiencia en términos generales ha sido muy buena, yo no tengo como una mala experiencia de compra por internet" (Botero, comunicación personal, 2018). Así como la suficiencia de la información ofrecida por las plataformas: "también que tenga la mayor información posible sobre el sitio, quiénes son, dónde están, la historia de la compañía si es posible, qué otros productos tienen, que tengan reviews de clientes, que tengan reviews de productos", (Botero, comunicación personal, 2018). Además, tratamiento de los datos personales:

No pues la página tiene mi dirección, mi teléfono, y pues mi nombre y eso. Hasta donde entiendo la página no usa estos datos hasta que yo no le dé comprar al artículo, entonces pues no creo que la información que pide es la suficiente para que uno se le haga efectiva la compra (Villanueva, comunicación personal, 2018).

Otro eje de indagación identificado es el riesgo percibido, donde los sujetos manifiestan su preocupación principalmente en la entrega de su información personal:

Yo creo que los riesgos son altos, porque tú tienes que meter los datos por ejemplo tu dirección, digamos cuando uno tiene que pagar no por efectivo sino con tarjeta pues peor, porque tú tienes que meter toda la información de tu tarjeta, la clave, o sea no, yo creo que el riesgo que tú tienes cuando compras con tarjeta es muy grande (Sepúlveda, comunicación personal, 2018). 
O las características del producto presentado en la plataforma respecto al que se espera recibir: "pues uno siempre al inicio le da a uno miedo que el producto no sea igual a las descripciones" (Mahecha, comunicación personal, 2018).

Con respecto al eje de seguridad percibida, los sujetos señalan la necesidad de seguridad en la conexión y en el tipo de dispositivo utilizado: "un portátil y que tenga red WiFi, obviamente no es cualquier red, no me meto a café internet ni nada de eso" (Mahecha, comunicación personal, 2018). "No utilizo ni el celular ni la tablet para hacer compras, son muy pocas veces, por ahí una vez no más lo he hecho, me gusta más en equipo, computador, computador de mesa o un portátil" (Mahecha, comunicación personal, 2018).

Adicionalmente, algunos insisten en el temor de que el producto comprado no corresponda con el visualizado en la plataforma:

No han sido positivas en su mayoría, porque yo no he comprado mucho por internet o sea todavía tengo como el miedo que el producto me salga malo o que yo pague y no me manden nada o que simplemente no sea de la calidad (Ferrer, comunicación personal, 2018).

Por otra parte, con respecto a privacidad percibida, si bien algunos sujetos manifiestan una inconformidad respecto a la información solicitada por estas plataformas, son conscientes de que la mayoría de estas las solicitan por requisitos y están dispuestos a entregarla: "Pues yo sí entregaría información demográfica, edad, de pronto algo de los gustos, pues casi todas preguntan los mismo" (Roa, comunicación personal, 2018). En el caso de que la plataforma solicite demasiada información, se convierte en un riesgo percibido para el consumidor en línea: "me toca... si me piden muchos datos personales para validar, no me interesa y dejo ahí, o sea no hago todo el proceso de compra, porque es muy tedioso, porque siento que están invadiendo mi privacidad" (Ferrer, comunicación personal, 2018).

En relación con la reputación percibida, los sujetos manifiestan la importancia de las opiniones dejadas por otros comparadores:

La calificación del vendedor es una de las que me pone, me tiene al tanto de eso, y los comentarios; casi siempre leo los comentarios o las preguntas, la forma en que el 
vendedor también responde las preguntas que le hacen y eso. Eso es lo básico para mí y la calificación del vendedor (Villanueva, comunicación personal, 2018).

Además de su experiencia: "como yo le digo, yo siempre trato de ingresar a páginas o lugares que sean conocidos, y que alguien ya haya tenido su experiencia al comprar", (Mahecha, comunicación personal, 2018). "Sí, reviso el historial de compras de las otras personas", (Mahecha, comunicación personal, 2018).

Así mismo, el reconocimiento de la marca genera una mejor percepción de la reputación de algunas plataformas:

El respaldo que tiene la aplicación, por ejemplo, Amazon, la calidad que siempre me ha salido muy buena e... y no solamente eso, también busco marca, sí, entonces yo primero hago una selección, miro, sé exactamente qué es lo que quiero (Roa, comunicación personal, 2018).

Esto, sumando a la importancia dada a los comentarios positivos:

Entonces digamos que si la mayoría de comentarios son muy positivos, y por ahí uno o dos que no son buenos, pues de pronto no es un motivo para que uno se cohíba de hacer cierta compra, a veces las compras que salen mal, con conocimiento de causa te lo digo, no son culpa del vendedor como tal sino a veces son culpa de las empresas de paquetería que le dan mal uso a los paquetes si se rompió algo (Rojas, comunicación personal, 2018).

En el eje de utilidad percibida se evidencia la importancia dada por los sujetos en cuanto a la rapidez:

La rapidez y facilidad, que yo puedo hacer la compra desde mi celular, puedo estar en cualquier lugar como el parque, la casa, la universidad, en cualquier parte, y saco el celular y ya puedo hacer una compra virtual, entonces yo creo que la compra virtual se hace es por la facilidad y rapidez (Sepúlveda, comunicación personal, 2018). 
Por otra parte, el interés por reducir el tiempo en los procesos de compra y las transacciones:

La uso por dos razones principales: uno porque físicamente me da mamera ir a buscar a las tiendas, entonces lo hice mucho tiempo y se gasta demasiado tiempo, las facilidades que te ofrece la red es que tú filtras inmediatamente lo que quieres, entonces en una plataforma por ejemplo como Aliexpress, como Amazon, como Groupon misma. Pero para lo que son vueltas o transacciones o el uso para acortar tiempo y hacer las cosas más fáciles es muy bueno (Villanueva, comunicación personal, 2018).

Finalmente, el atractivo de descuentos y variedad de productos ofrecidos por las plataformas es ampliamente valorado: "lo que te digo, uno se da cuenta que hay bastantes descuentos, entonces uno compara los precios de esa plataforma con los del mercado real y uno se da cuenta que sí es un buen descuento" (Rojas, comunicación personal, 2018).

Otros mencionan la importancia de que la plataforma de pago sea reconocida:

Qué información estoy dispuesto a dar, únicamente: correo electrónico, número de celular, el login, es suficiente no necesitan un perfil, porque el perfil te lo da del hábito de compra, o sea no necesitas preguntárselo al cliente, pues qué información es necesaria, pues claramente la tarjeta de crédito, pero tiene que venir avalado por una plataforma internacional como Payu o debe venir avalado por un PSE (Devia, comunicación personal, 2018).

Igualmente, un diseño apropiado y profesional genera una percepción de seguridad hacia la página: "las páginas que son pues que son más o menos estafosas, no suelen tener diseños tan cuidados, porque tener un diseño cuidado es una inversión importante" (Botero, comunicación personal, 2018).

Referente a la calidad del servicio percibido, los sujetos presentan interés por establecer una comunicación con el vendedor y el seguimiento que este les hace hasta la entrega del producto:

Una vez me pasó con una página de vestidos de baño en Facebook y nada más le di seguir y de una vez me mandaron un mensaje en Messenger y decía "Bienvenida a la 
página, por aquí podrás encontrar diferentes productos, nuestras promociones son estas... si estás interesado en algo por favor respondenos el mensaje". Eso me parece súper chévere, que yo acabo de seguir a una página y automáticamente me estaba llegando el mensaje, y yo percibí que ellos realmente estaban interesados en que yo les hiciera una compra (Sepúlveda, comunicación personal, 2018).

Además, también es importante el trato del vendedor en el servicio prestado: "Algo que me motivaría a seguir comprando sería pues el servicio, es importante, el servicio que te da el vendedor es vital, que te trate bien, que esté pendiente, que se preocupe por todo el proceso de compra" (Villanueva, comunicación personal, 2018). Así como también la posibilidad de que el cliente evalúe o califique el proceso de compra luego de recibido el producto: "Ellos le envían a uno un mensaje para que uno de la calificación, de la calificación del producto, diga que le pareció, como estuvo, cómo llego" (Villanueva, comunicación personal, 2018). También es relevante el acompañamiento previo a la compra para la toma de decisiones más confiada y segura para el cliente: "en algunos casos, entro específicamente a buscar lo que necesito. En otros, pues la página se encarga de enviarme al correo, propagandas, descuentos y eso, entonces ahí veo ya si me sirve, no me sirve" (Villanueva, comunicación personal, 2018).

Relativo a la calidad de la información percibida, los entrevistados mencionan la relevancia y disponibilidad de una información completa en la página web:

A mí me da mucho mal genio cuando las páginas ofrecen cosas y no ponen el precio, y muchas páginas hacen eso, digamos ponen una blusa súper linda y no dicen el precio, entonces a ti te toca escribir para que te den el precio del producto, entonces ¿Cuál es la necesidad de no dejar el precio ahí? (Sepúlveda, comunicación personal, 2018). Que tenga bastante información sobre el producto, o sea como, entre más, mejor, si me especifican el peso o el tamaño o sea entre más me pueden especificar mejor, porque eso me permite a mi tomar una decisión mucho más informada (Botero, comunicación personal, 2018).

Por otro lado, se manifiesta que elementos como usabilidad, claridad, manejo intuitivo y sencillez son valorados dentro de la calidad del diseño percibido: "sobre todo para el tema digamos de las plataformas hay dos elementos que para mí son clave: uno es la usabilidad, 
es decir, que sea una plataforma que sea intuitiva que no tiene tanta información que diga claritico". "Me parece que es una plataforma sencilla, Incluso cuando ya compras el pasaje, ellos te dan unos tips..." (Ferrer, comunicación personal, 2018). Así como la percepción de calidad en páginas con diseño profesional: "que la página esté expresamente como diseñada por alguien que sepa de diseño web, que eso es muy evidente cuando uno compara una página hecha por alguien que no sabe, a una página hecha por alguien que sí sabe" (Botero, comunicación personal, 2018).

Algunos sujetos también consideran la necesidad de que la plataforma cuente con sistemas de calidad que les permita tener confianza de los procedimientos frente a cualquier inconveniente en los casos de compra, distribución y devolución del producto:

"Tuve la experiencia de comprar unos tenis y el tallaje me quedó grande, entonces tuve que hacer la devolución, pero no fue con Adidas, sino fue directamente con Falabella, entonces Falabella hizo la devolución y me tocó hacer la devolución en la misma tienda, entonces en ese caso hice la compra por internet y me hicieron en la misma tienda; entonces no hubo problema por ese lado" (Mahecha, comunicación personal, 2018). "Si tú tienes una política de servicio, que la política de servicio se cumpla, cuando no se cumple también es bueno que te lo digan".

Los entrevistados indicaron que la satisfacción final sobre el proceso de compra de una transacción en línea ha sido generalmente una experiencia positiva, y sobre la cual no presentan comentarios negativos, si no por el contrario argumentan que esta satisfacción se logra en la medida en que, no solamente los atributos del sitio web generan confianza, si no la necesidad de informarse debidamente sobre el producto el sitio web y el proveedor en línea: "Pues digamos a modo personal todas las compras virtuales que yo he realizado han sido muy buenas, siempre y cuando uno siga todos los parámetros" (Rojas, comunicación personal, 2018) Además, manifiestan que en la medida que se cumpla con los procedimientos requeridos para la compra en el sitio web, no se tendrá ninguna experiencia negativa:

No pues realmente ha sido muy bien, como yo nunca he tenido problemas, de ningún tipo, no he tenido problemas con las tarjetas de crédito, no he tenido problemas como... con que me lleguen productos diferentes a las descripciones, yo siempre leo 
muy cuidadosamente los reviews de otros compradores veo fotos (Botero, comunicación personal, 2018).

Con relación al eje de actitud, los entrevistados presentan posiciones favorables y desfavorables frente a compra en línea. De un lado, consideran la importancia del respaldo y garantía del proveedor en línea:

Quizá en toda mi historia de compras he tenido una o dos veces que el producto no me ha llegado, pero la ventaja que tiene, digamos, la plataforma en la que compro particularmente, en las dos que me ha pasado, es que te reintegran el dinero, entonces te abonan un crédito y pues tú desde ahí (Devia, comunicación personal, 2018).

Por otra parte, se identifican actitudes negativas o de desconfianza en las cuales algunos de los entrevistados manifiestan temor por la transacción en línea:

Porque yo no he comprado mucho por internet o sea todavía tengo como el miedo que el producto me salga malo o que yo pague y no me manden nada ¿sí? O si no que simplemente no sea de la calidad o sí sería eso (Ferrer, comunicación personal, 2018).

Adicionalmente, no se debe desestimar la importancia de aspectos como el precio, como la variedad de oferta y promociones que incentivan la compra en línea:

Pues utilizado AliExpress, también para comprar ropa y para comprar como una cosa para las uñas para echarme aceite en las uñas, que, pues me sale también mucho más barato, y pues con la ropa también lo mismo o sea me sale más barato, pero, además, tiene una mucha mayor oferta, de lo que hay acá (Botero, comunicación personal, 2018).

La intención de compra se constituye en otra variable analizada en los entrevistados, que plantean la importancia de criterios como diseño, reputación, calidad y precio percibidos como condicionantes para comprar en línea por primera vez:

El respaldo que tiene la aplicación, por ejemplo Amazon, la calidad que siempre me ha salido muy buena e... y no solamente eso, también busco marca, sí... entonces yo primero hago una selección, miro, sé exactamente qué es lo que quiero busco qué 
productos me atienden esa necesidad y con respecto a eso e... busco cuál es la mejor marca en esa categoría, y ya al finalizar sí busco el producto y trato de mirar que hayan varias ofertas y busco la más económica (Roa, comunicación personal, 2018).

Así mismo, la suficiencia de la información ofrecida por el proveedor en línea y los tiempos y procesos de entrega del producto:

Pues páginas más reconocidas, Falabella, de pronto Alkosto, hay una página nueva que me gusta que se llama AliExpress, que trae productos de China o Japón realmente no sé muy bien, y la he comprado a través de esa página y me ha ido muy bien, lo malo es que se demora de 20 días a 1 mes en traer el producto (Mahecha, comunicación personal, 2018).

En cuanto a la intención de re-compra, los entrevistados evalúan su experiencia: "Por la seguridad y por la garantía que me ha dado la página” (Villanueva, comunicación personal, 2018). Además de la utilidad de dejar sus comentarios para mejoramiento del servicio por parte del proveedor en línea en beneficio de otros consumidores:

Pues seguramente muchas personas tienen la misma duda y también necesitan tener la tranquilidad de que es una página confiable, entonces entro y obviamente hago mi apreciación por tal motivo, pues también por esa experiencia que yo cuento le va a servir a alguien más (Mahecha, comunicación personal, 2018).

Sin embargo, plantea que la primera experiencia es determinante en la decisión de recompra:

La seguridad de que voy a recibir lo que el proveedor me está indicando, y la confiabilidad de la página, que hice el pago una vez, pues seguramente es una página segura y confiable, pues si me funcionó una vez me puede funcionar dos veces, teniendo en cuenta los parámetros que tomo como tal.

La intención de uso de una página web se comprende desde la utilidad, funcionalidad y rapidez provistas por un consumidor en línea, que ofrece la información necesaria para generar la confianza de su uso: "la facilidad de encontrar el producto que uno quiere, la 
rapidez y la facilidad de encontrarlo" (Mahecha, comunicación personal, 2018). Por otra parte, se tiene en cuenta el conocimiento previo y reputación del proveedor en línea, ya sea por su marca o por las recomendaciones obtenidas del entorno:

Digamos de lo que te decía las que más utilizo son Rappi y Avianca, digamos que frente a mi experiencia con Rappi fue buena porque te llevan la comida, te dicen el tiempo en el que te llega, y pues efectivamente te llega (Sepúlveda, comunicación personal, 2018).

Sin mencionar la experiencia acumulada en el uso de la página web: "nunca he tenido problema, ni tampoco he recibido el producto que no esperaba o algo así, siempre ha sido lo que he pedido, como lo quería y todo" (Villanueva, comunicación personal, 2018).

La lealtad es considerada por parte de los entrevistados por medio de la consistencia de los valores del producto adquirido: como actitudes, acompañamiento, tiempo y utilidad, entregados por parte del proveedor en línea, para mantener asegurada la confianza y el grado de compromiso del consumidor en línea:

Que también me parece súper interesante de las plataformas, que te premian un poco en fidelidad, pues intentan fidelizarte también y te mandan ofertas que todo el tiempo te están entregando información o que entre las estrategias de fidelización te colocan cosas como: no nos hemos olvidado de ti cupón del 20\% (Devia, comunicación personal, 2018).

Esta lealtad es percibida los entrevistados como la preocupación del proveedor en línea durante el proceso de compra: "Algo que me motivaría a seguir comprando sería pues el servicio, es importante el servicio que te da el vendedor es vital, que te trate bien, que esté pendiente, que se preocupe por todo el proceso de compra" (Rojas, comunicación personal, 2018).

Por otra parte, en lo relacionado con los hábitos del consumidor en línea, se identificó entre los entrevistados la importancia del ahorro monetario percibido desde la oferta y promociones, adicional al ahorro de tiempo, al evitar desplazamientos que agilizan el proceso de compra: 
Bueno pues los tiquetes de avión, pues porque que pereza ir hasta Avianca, pues si yo puedo buscarlo por Internet mejor porque ya me di cuenta de que no es tan complicado, entonces digamos que la compra de tiquetes por Internet me parece muy buena (Sepúlveda, comunicación personal, 2018).

Pues realmente término medio, pues obviamente me ahorra mucho el desplazarme de un lado a otro, y a veces encuentro las cosas más económicas (Mahecha, comunicación personal, 2018).

Así mismo, la conveniencia representa uno de los atributos que ofrecen las plataformas e-commerce, dentro de las prácticas de los consumidores en línea:

En Rappi por ejemplo solamente el día de antes de ayer hice una compra también de comida rápida, que me llegó a la casa y fue muy rápido muy útil, lo mismo lo hecho con domicilios.com, entonces sí considero que para mí es indispensable tenerlas... esas plataformas digitales (Roa, comunicación personal, 2018).

Con el análisis de los hábitos, los entrevistados hacen observaciones relacionadas también con el valor que les representa una transacción en línea por la utilidad percibida, el costo y el tiempo requerido para hacerla:

Bueno beneficios: Rapidez, uno ya sabe a lo que va, lo que espera del producto; economía, porque pues es mucho más barato; no estás perdiendo tiempo porque todo lo estás haciendo desde el lugar donde estés; creatividad, porque casi siempre esos productos no los encuentras en los negocios físicos y casi siempre ves es la camiseta estampada de una banda y eso no lo hay en cualquier lugar entonces es como el diferencial también (Ferrer, comunicación personal, 2018).

Pues realmente término medio, pues obviamente me ahorra mucho el desplazarme de un lado a otro, y a veces encuentro las cosas más económicas (Mahecha, comunicación personal, 2018). El costo y que no tengo que salir de mi casa o de mi oficina para comprar (Mahecha, comunicación personal, 2018).

Los entrevistados refieren que dentro sus hábitos, a pesar de los atributos, la información y la seguridad percibidas son relevantes para la toma decisiones en el consumo en línea, las características y acceso a la web constituyen en el punto de partida para cerrar 
la transacción mediante el uso de cualquier dispositivo: "no utilizo ni el celular ni la tablet para hacer compras, son muy pocas veces, por ahí una vez no más lo he hecho, me gusta más en equipo, computador, computador de mesa o un portátil" (Mahecha, comunicación personal, 2018). "Un portátil y que tenga red WiFi, obviamente no es cualquier red, no me meto a café internet ni nada de eso" (Mahecha, comunicación personal, 2018).

Finalmente, se plantea que los aspectos de seguridad y disposición para la construcción de la confianza en línea, no solamente se encuentran representados en las características del sitio web y en el manejo de la información requerida por los proveedores en línea, sino, también en el elementos que permitan la verificación de la información, por medio de historiales de comentarios generados por otros consumidores y de la comparación con otras plataformas que ofrecen los mismos productos:

He mirado la página, sus características, y obviamente vuelvo y miro a las dos o tres semanas después y miro mecanismos, si ha generado cambios o algo, y consulto a través de otros computadores a ver la página obviamente tiene otra ruta o algo, que no me genere colores o imágenes que no sean, qué no me pregunten información personal de entrada (Mahecha, comunicación personal, 2018).

\subsection{Redes semánticas}

De acuerdo con lo anterior, los resultados de las entrevistas a profundidad aplicadas en la investigación demuestran la importancia en la relación de las necesidades identificadas de los consumidores en línea en la categoría de confianza en línea, como mediador en las prácticas de este tipo de consumo. Esta confianza comprende tres atributos fundamentales: la integridad, la habilidad y la benevolencia (Kim \& Peterson, 2017), explicados desde cada uno de los ejes de indagación que determinan el grado de satisfacción final del consumidor en las transacciones en línea. A continuación, se presenta la red semántica de necesidades (definida como confianza la línea como categoría) que refleja la relación manifestada por los entrevistados en los resultados obtenidos. 
Figura 1. Red semántica de necesidades consumidores en línea programa Atlas.ti

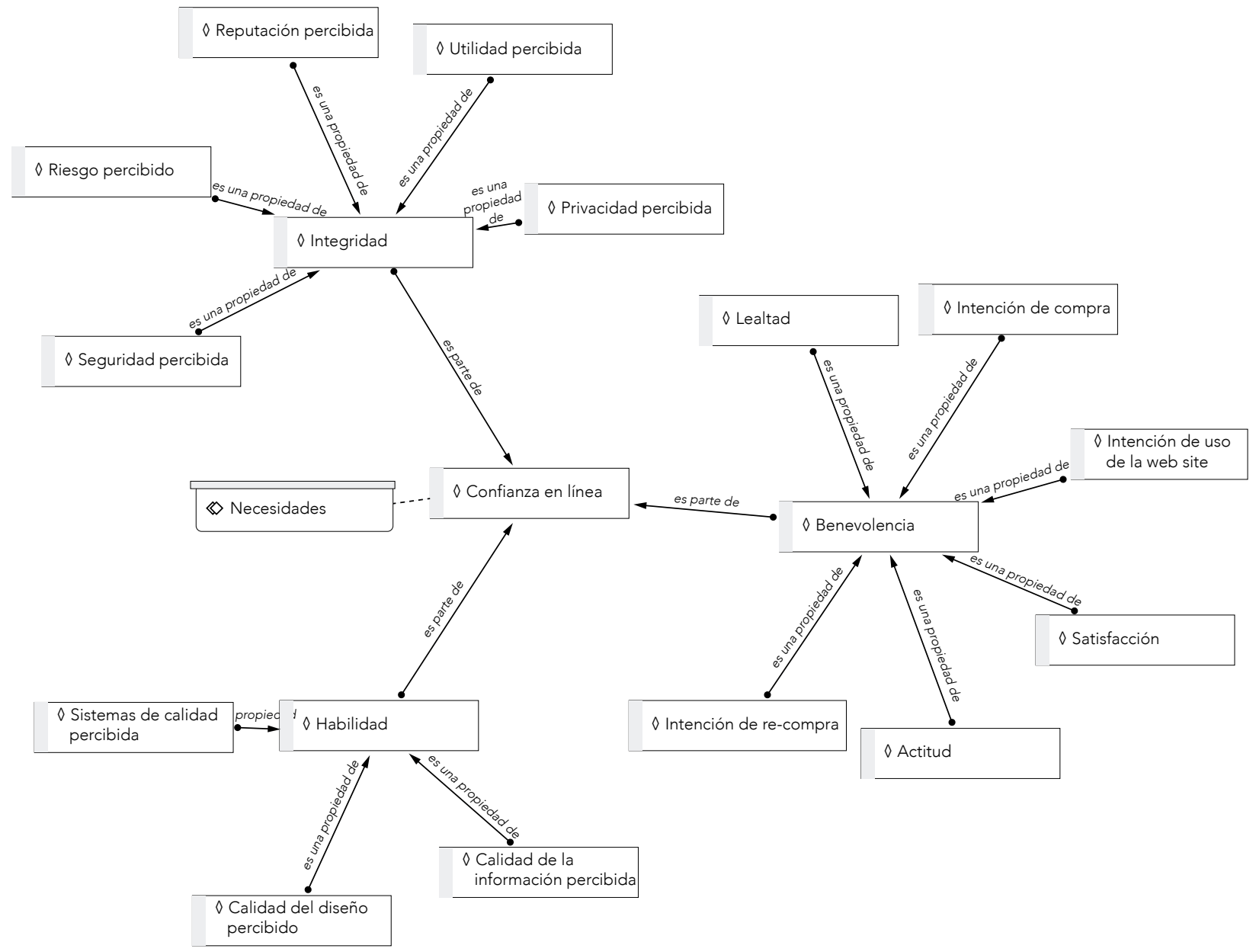

Fuente: elaboración propia con base en resultados obtenidos por los autores (2018).

\section{Discusión}

Los resultados obtenidos de la investigación indican que el grupo objeto de estudio comprende la disposición de confianza en línea a partir de cuatro aspectos fundamentales: un entorno virtual adecuado de la página web, una información suficiente y clara para la toma de decisiones, la seguridad en el tratamiento de los datos suministrados y una buena valoración por otros clientes por medio de los comentarios registrados, que contrastan con lo planteado por Fukuyama (1995), respecto a la confianza comprendida a partir del contexto cultural y la experiencia de vida, sobre los cuales los sujetos manifiestan, en general, haber tenido buenas experiencias. 
Frente a la privacidad percibida, se identificó que la cantidad de información requerida osolicitada por las plataformas de e-commerce influyen sobre la decisión final de compra, existe un tipo de información personal que el consumidor en-línea percibe como necesaria y relevante para un proceso regular de compra como: nombre, identificación, dirección, correo electrónico y medio de pago, sin embargo, cuando la plataforma solicita información como edad, género, fecha de nacimiento, pasatiempos, etc., algunos entrevistados la perciben como invasiva, llegando inclusive a no cerrar el proceso de compra. Como lo mencionado por Casaló, Flavián, y Guinalíu (2007), sobre la percepción de los requerimientos legales y las buenas prácticas, que existen para el manejo de la información personal, a partir de los cuales, los consumidores no desean asumir riegos que afecten la seguridad de sus datos y exigen del e-commerce, esa seguridad.

Referente a la reputación percibida, los entrevistados plantearon la importancia del historial de comentarios sobre las experiencias de compra, registrados por otros clientes, adicional, al reconocimiento de la marca de la tienda en línea, que se comprende como requisito mínimo para la toma de decisión, sin embargo, también se identificó un cierto grado de duda sobre la validez de aquellos comentarios que son negativos y sobre los cuales la tienda da respuesta, como lo señalado por Doney y Cannon (1997), referente a la percepción sobre si el canal es honesto y preocupado por sus consumidores, donde las personas confían en la elección del proveedor actual y en las futuras intenciones de compras.

Con respecto al riesgo percibido, como lo mencionan Kim, Ferrim y Rao (2008), sobre la incertidumbre potencial de los resultados negativos de una transacción en línea, los entrevistados señalan dos aspectos importantes: de un lado, el riesgo por la entrega de la información financiera y de otro, la incertidumbre de que el producto real a recibir corresponda con las especificaciones presentadas en la página.

Muy relacionado con el diseño percibido, el riesgo percibido y la disposición de confianza, se encuentra la privacidad percibida, dado el uso y manejo de la información personal por medio de las compras en línea. Como lo señalan McKnight y Norman (2001, citados por Kim \& Peterson, 2017), la perspectiva de seguridad ha sido definida como la garantía legal y tecnológica percibida por el consumidor. Así, los entrevistados indican la importancia de que el diseño de la página, los requerimientos legales, y en general la 
información solicitada por el sitio web, debe ir acompañados de mecanismos de seguridad apropiados para la protección de los datos del consumidor como de la misma empresa (Chen \& Barnes (2007).

Así mismo, como lo expuesto por Davis (1989, citado por Kim \& Peterson, 2017), los consumidores otorgan el atributo de utilidad a la facilidad tecnológica ofrecida por el sitio web en el proceso de transacción. Los entrevistados señalan ampliamente la rapidez como sinónimo de utilidad percibida, en la medida en que se les facilite realizar las transacciones desde cualquier dispositivo electrónico, como también, desde cualquier lugar

Por otro lado, la evaluación de la calidad de la interacción con la plataforma por parte del consumidor demuestra el grado de satisfacción subjetiva sobre el servicio, que incluye desde el instante en que se hace contacto con la plataforma, hasta la recepción física del producto, y en la cual el empresario debe hacer seguimiento, gestión y generar la debida confianza para que el consumidor en línea se sienta respaldado. Brown y Jayakody (2009, citados por Kim \& Peterson, 2017) plantean la apreciación de los consumidores sobre los esfuerzos generados por las plataformas para garantizar la integridad de todo el proceso, como un referente de calidad de servicio percibido.

Otro de los ejes de indagación que son muy valorados por los entrevistados, es la calidad del diseño del sitio web, el cual debe guardar un cierto balance entre la usabilidad, la claridad de la información, componentes intuitivos y la estética del entorno apropiada y profesional como factores relevantes en la construcción de la confianza en línea y, por ende, en la decisión de compra. Bart, Venkatesh, Fareen y Glen (2005, citado por Kim \& Peterson, 2017) exponen que una apariencia visual de la página web demuestra la capacidad y profesionalismo del vendedor en línea, que debe generar la misma confianza en línea.

Los entrevistados consideran que, de forma tácita, los sistemas de calidad deben estar implícitos dentro de las mismas transacciones en línea y que, a pesar de que el diseño, la utilidad, la privacidad, la información y la reputación percibidas son importantes para la toma de decisiones. Las empresas deben garantizar un sistema que le asegure al consumidor en línea la entrega de su producto en las condiciones y tiempos acordados, es decir sistemas de calidad que integren la compra, la entrega y el servicio post venta. Aladwani y Prashant (2002, citado por Kim \& Peterson, 2017) están de acuerdo con que 
las características funcionales y técnicas de un sistema de información en una plataforma de e-commerce deben estar asociadas con la confiabilidad, flexibilidad, accesibilidad y oportunidad para la construcción de la confianza del consumidor en línea.

Frente al eje de actitud, los entrevistados exponen claramente múltiples beneficios que ofrece la compra en línea, especialmente por las alternativas de oferta y precio, así como también manifiestan temores inherentes al producto, manejo de datos personales y pago. Teo, Thompson y Jing Liu (2007, citados por Kim \& Peterson, 2017) corroboran que las características de confiabilidad de un proveedor en línea como capacidades, benevolencia e integridad, definen la actitud positiva o negativa de los consumidores

La intención de compra, como otro elemento de análisis de los hábitos, necesidades y prácticas de consumo de las plataformas en línea, se encuentran definidas por la probabilidad que identifican los consumidores, basados en una serie de elementos provistos por el proveedor en línea por medio de la página web y que logran incrementar los niveles de confianza para la compra de un producto (Kim, Ferrin \& Rau, 2009), como es el caso de lo planteado por los entrevistados durante la investigación sobre precio, ofertas y garantía de entrega del producto. El diseño, la información, la reputación y la utilidad del proveedor en línea por medio de la página Web se consideran como elementos mínimos implícitos.

El compromiso voluntario del consumidor para la recompra de un producto, parte la percepción que se forma a partir de la experiencia acumulada por el uso de la plataforma en línea, y de la satisfacción obtenida de esas experiencias. Chiu, Meng-Hsiang, Hsiangchu y Chu-Ming (2012, citados por Kim \& Peterson, 2017) señalan que la construcción de más altos niveles de confianza, a partir de la experiencia acumulativa, es lo que motiva al consumidor a obtener productos de la misma fuente o proveedor.

En relación con la intención de uso de la página web, el proveedor en línea debe aprovechar las ventajas generadas por la experiencia acumulada por su uso, así como las estrategias de marketing que incentivan la recompra y reducen la probabilidad de conductas de compra de oportunidad ofrecidas por otros proveedores, como lo señalado por Gefen, Karahanna y Straub (2003), sobre la comprensión de la conducta del consumidor en línea por medio del fomento de la confianza en el uso de la web. 
La lealtad parte de la confianza asegurada y del grado de compromiso sobre la marca, manifestada por una actitud positiva en el número de visitas, número de compras e interacción con la plataforma, como lo exponen Cyr, Hassanein, Head e Ivanov (2007, citados por Kim \& Peterson, 2017), frente a lo cual los entrevistados valoran los aspectos y estrategias que utilizan los proveedores en línea para mantener la confianza como las información, las ofertas, el acompañamiento y el tiempo de entrega en el proceso de compra en línea, que genera una lealtad.

En cuanto a la satisfacción, el número de experiencias o encuentros positivos del consumidor en línea en el sitio web permiten el fortalecimiento de su confianza, y por ende lo conduce a una satisfactoria experiencia de compra. La información que previamente es investigada sobre el producto por parte del consumidor asegura una mejor satisfacción del producto adquirido, como lo señalan Chen y Chow (2012) y Anderson y Srivasan (2003), (citados por Kim \& Peterson, 2017).

Todo lo anterior presenta la descripción de las percepciones de los entrevistados frente a la confianza en línea y las características que establecen las prácticas de consumo. De igual manera, los hábitos que constituye el perfil del consumidor en línea, definidos desde cuatro dimensiones (tecnológica, económica, social y legal) (Kim \& Peterson, 2017), se evidencian desde lo planteado sobre su conveniencia, valor, conectividad y protección (Kucuk \& Krishnamurthy, 2007). Recordando lo señalado por Bordieu (2000, citado por Kucuk \& Krishnamurthy, 2007) sobre los hábitos como un sistema de estructuras: "sistemas de disposiciones duraderas, estructuras estructuradas predispuestas a funcionar como estructuras estructurantes, es decir, en tanto que principios de generación y de estructuración de prácticas y representaciones", las prácticas de consumo y las representaciones que los individuos tienen ellas constituyen el enfoque de otras representaciones o prácticas subsecuentes, creando valor para el consumidor en línea.

En este orden de ideas, de acuerdo con lo mencionado por Kucuk y Krishnamurthy (2007), la conveniencia evidencia que le beneficio para el consumidor en línea, desde la dimensión tecnológica, está representada por la conveniencia generada a partir del proveedor, la movilidad superior y la accesibilidad en cualquier momento y desde cualquier lugar (24 horas, 7 días a la semana). De igual forma, los entrevistados manifiestan desde la 
dimensión tecnológica los valores percibidos por el acceso a productos más económicos y desde una amplia variedad de opciones de oferta por medio de la web.

Adicionalmente, la conectividad como un beneficio desde la dimensión social, le permite al consumidor en línea el acceso a redes sociales, comunidades y expertos para contrastar la información presentada, por las plataformas en línea para la toma de decisiones de compra. Esto se encuentra muy relacionado con la dimensión legal, que caracteriza el beneficio de la protección percibida por los proveedores en línea por medio del cumplimiento de las normas legales y la protección de los datos entregados por el consumidor en línea en las respectivas plataformas e-commerce, al momento de la transacción.

\section{Conclusiones}

El análisis de los resultados obtenidos, frente a las entrevistas a profundidad, permitieron contrastar los antecedentes teóricos sobre el consumo en línea e identificar la relación de los hábitos, necesidades y prácticas de consumo en plataformas digitales de e-commerce. Con respecto a la confianza en línea, se evidenció el valor establecido por los consumidores a aspectos como la información suministrada por el sitio web, la calidad del servicio posterior a la transacción en línea, la seguridad percibida y la utilidad en el uso de las plataformas en línea para la toma de decisiones de compra.

Es importante resaltar que esta decisión está íntimamente ligada con los referentes de otros sitios web y aquellos suministrados por los historiales de comentarios de otros consumidores, que, aunque no son definitivos para la toma de decisión, sí influyen ampliamente en esta. La intención de recompra se encuentra determinada por la experiencia misma y es evidenciada en la búsqueda permanente de información por parte de los consumidores para el perfeccionamiento de su propia experiencia. La lealtad verdadera está altamente relacionada con la satisfacción y actitud de dicha experiencia.

La conjunción de todas estas variables, contrastadas en el entorno local, demostraron gracias a la investigación, que las prácticas de consumo en línea corresponden a lo planteado teóricamente desde los antecedentes y consecuencias que permiten la construcción 
de relaciones confiables entre el consumidor en línea y los proveedores en línea, desde la disciplina del mercadeo y las prácticas de consumo en un escenario no virtual están determinadas por variables como precio, calidad, accesibilidad y reputación de las marcas. En los escenarios de las plataformas e-commerce, los consumidores adicionalmente requieren de aspectos que consoliden una verdadera confianza, experiencia y satisfacción para la toma de decisiones de compra y consumo.

\section{Referencias}

ANDI (2018). Colombia hizo clic en materia digital: Bruce Mac Master. Asociación Nacional de Empresarios de Colombia. Recuperado de http://www.andi.com.co/Home/Noticia/1074colombia-hizo-clic-en-materia-digital-br

Bautista, C. (2011). Proceso de la investigación cualitativa: Epistemología, metodología y aplicaciones. Bogotá: Manual Moderno.

Casaló, L., Flavián, C. \& Guinalíu, M. (2007). The impact of participation in virtual brand communities on consumer trust and loyalty: The case of free software. Online information review, 31(6), 775-792.

Chen, Y. H. \& Barnes, S. (2007). Initial trust and online buyer behaviour. Industrial management \& data systems, 107(1), 21-36.

Comisión de Regulación de Comunicaciones -(СRC) (2018). Reporte de Industria Sector TIC 2017. Recuperado de https://www.crcom.gov.co/recursos_user/reporteindustria2017.pdf

Consejo Privado de Competitividad (2018). Informe Nacional de Competitividad 2018-2019. Recuperado de https://compite.com.co/wp-content/uploads/2018/10/CPC_INC_20182019_Web.pdf

Davis, F. D. (1989). Perceived usefulness, perceived ease of use, and user acceptance of information technology. MIs Quarterly, 13(3), 319-340.

Doney, P. M. \& Cannon, J. P. (1997). An examination of the nature of trust in buyer-seller relationships. Journal of marketing, 61(2), 35-51.

Fukuyama, F. (1995). Trust: The social virtues and the creation of prosperity (Vol. 99). New York, NY: Free press.

Gefen, D., Karahanna, E. \& Straub, D. W. (2003). Trust and там in online shopping: an integrated model. MIs Quarterly, 27(1), 51-90. 
Kim, D., Ferrin, D. \& Rao, H. (2008). Trust-based consumer decision-making model in electronic commerce. The role of trust, perceived risk, and their antecedents. Decision Support Systems, 2(44), 544-564.

Kim, Y. \& Peterson, R. (2017). A Meta-analysis of Online Trust Relationships in E-commerce. Journal of Interactive Marketing, (38), 44-54.

Kucuk, S. U. \& Krishnamurthy, S. (2007). An analysis of consumer power on the Internet. Technovation, $27(1-2)$, 47-56.

Li, Q., Quansheng, W. \& Lin, Z. (2017). Effects of consumer visit to online community and product channel on local sales of large consumer goods: Evidence from real estate industry. Journal of Strategics Information Systems, (27), 191-204.

Martínez-López, F. J., Anaya-Sánchez, R., Molinillo, S., Aguilar-Illescas, R. \& Esteban-Millat, I. (2017). Consumer engagement in an online brand community. Electronic Commerce Research and Applications, (23), 24-37.

McKnight, D., Cummings, L. \& Chervany, N. (1998). What trust means in e-commerce customer relationships: An interdisciplinary conceptual typology. International Journal of Electronic Commerce, 2(6), 35-59.

Pavlou, P. (2003). Consumer acceptance of electronic commerce: Integrating trust and risk with the technology acceptance model. International Journal of Electronic Commerce, 3(7), 69-103.

Thompson, S. A. \& Sinha, R. K. (2008). Brand communities and new product adoption: The influence and limits of oppositional loyalty. Journal of marketing, 72(6), 65-80.

World Economic Forum (2018). The Global Competitiveness Report 2018. Recuperado de http:// www3.weforum.org/docs/GCR2018/05FullReport/TheGlobalCompetitivenessReport2018. pdf. p. 163-165.

World Economic Forum (2019). Colombia asume el papel de líder mundial en la Cuarta Revolución Industrial y abre un centro de tecnología emergente en Medellín. Recuperado de https:// www.weforum.org/press/2019/04/colombia-asume-el-papel-de-lider-mundial-en-la-cuarta-revolucion-industrial-y-abre-un-centro-de-tecnologia-emergente-en-medellin/ 